\title{
The distribution of pyloric mucosa in partial gastrectomy specimens
}

\author{
A. C. B. DEAN AND M. K. MASON \\ From King's College Hospital and Medical School, London
}

EDITORIAL SYNOPSIS In a study of 117 partial gastrectomy specimens it is shown that all the pyloric mucosa had not been completely removed in $7.9 \%$ of gastrectomies for duodenal ulcer. In addition, a significant amount of pyloric mucosa was found to have been left behind at the original operation in five out of six patients who had a second gastrectomy for stomal ulcer, It is suggested that pyloric mucosa remaining in the proximal part of the stomach is responsible for maintaining acid secretion, which may result in the production of stomal ulcers.

There has been no instance in this series of a gastric ulcer developing in body mucosa. Women with gastric ulcers were found to have more pyloric mucosa than men with gastric ulcers.

Duodenal ulcer can be cured by any operation which diverts the gastric contents away from the duodenum, but such an operation must also reduce gastric secretion if stomal ulceration is to be avoided. Partial gastrectomy achieves this by removing some of the acid-secreting body mucosa together with the pyloric mucosa which produces the hormone gastrin, a powerful stimulant of gastric secretion. The importance of this 'hormonal phase' was realized comparatively late in the history of partial gastrectomy. Finsterer's operation of antral exclusion had become popular for cases where it was difficult to mobilize the first part of the duodenum (Finsterer and Cunha, 1931). It later became obvious that this operation, in which most of the pyloric antrum was left in continuity with the duodenum, was associated with a high incidence of stomal ulceration, indicating that the antrum was responsible for maintaining a high level of acid secretion. This led to a re-examination of the earlier work of Edkins and Tweedy (1909) on the secretion of an antral hormone. Eventually Gregory and Ivy (1941) confirmed the existence of the hormone gastrin and the role of the antrum in its production. Gastrin has since been extracted from antral mucosa in a pure form and its physiological properties investigated (Harper, 1946; Gregory and (Tracy, 1961). As a result of this discovery surgeons have taken particular care to remove all the pyloric mucosa at the duodenal end of the stomach. It is surprising that little attention has been paid to the possibility that pyloric mucosa might be left behind in the stomach remnant, either because an inade- quate gastrectomy had been done, or because the pyloric mucosa extended unusually far up the lesser curve.

We have therefore set out to investigate the frequency with which pyloric mucosa is incompletely removed at partial gastrectomy. In addition, we have examined the sections from patients on whom a second gastric resection has been carried out because of stomal ulceration following partial gastrectomy, to discover whether pyloric mucosa had been left behind at the original operation.

\section{METHODS}

One hundred and seventeen stomachs removed by partial gastrectomy for either gastric or duodenal ulcer were used in this study which covered the years 1959-61 inclusive. All specimens were received in the fresh, unfixed condition within a few minutes of excision. They were opened along the greater curvature, pinned out flat on a piece of cork board with the mucosa upwards, and fixed in this way in formol-saline for a period of 24 to 48 hours. The stomachs were then unpinned and the lesser and greater curvatures were measured. The entire lesser curve and in some cases the greater curve as well was sectioned and examined using the 'Swiss roll' technique (Magnus, 1937).

The 'Swiss roll' was prepared as follows:-

A strip of stomach wat $1.0 \mathrm{~cm}$. wide was cut from the part to be examined. This was then rolled up with the mucosa outwards and tied with thread, forming a 'Swiss roll'. This was then processed, sectioned, and stained with haematoxylin and eosin in the usual way. By this means the entire lesser or greater curve was present on one slide.

The 'Swiss rolls' were examined microscopically and the junction of pyloric and body mucosa was marked. 
The extent of the pyloric mucosa from the pyloro-duodenal junction was then measured. Particular attention was focused on the extent of the pyloric mucosa along the lesser curve, and in all 117 cases this measurement was made. The length of body mucosa present along the remainder of the lesser curve was also measured.

In order to find out if the extent of pyloric mucosa could be assessed macroscopically with reasonable accuracy, additional measurements were made on 18 of these stomachs. The junction of pyloric and body mucosa was judged on the unfixed specimen along both the lesser and greater curvatures, and the distance from the pylorus measured. This was compared with the extent of pyloric mucosa measured microscopically in strips from the greater and lesser curves. These measurements were also used to map out the distribution of pyloric mucosa.

We have collected six patients who had undergone a previous gastrectomy and subsequently developed a stomal ulcer. A second gastric resection was performed and sections from these specimens were examined to see if pyloric mucosa had been left behind at the first operation.

\section{RESULTS}

DUODENAL ULCER Out of 63 gastrectomy specimens from patients with duodenal ulcers, there were five $(7.9 \%)$ which had pyloric mucosa at the upper end of the lesser curve (Table 1). In the other 58 stomachs a gradual transition from pyloric to body mucosa on the lesser curve was noted in $55 \%$. This transitional zone extended over an average distance of $1.0 \mathrm{~cm}$., but it varied from 0.5 to $5.0 \mathrm{~cm}$. The transition to body mucosa was beginning to appear in two of the five stomachs in which the line of gastrectomy was made through the pyloric mucosa. In the remaining three cases no body mucosa was present on the lesser curve.

A plan showing the distribution of the pyloric mucosa was obtained by measurements on the 18 stomachs in which strips were also cut from the anterior and posterior walls and the greater curvature (Fig. 1). It can be seen that, on average, the pyloric mucosa extended for only $1.5 \mathrm{~cm}$. further up the lesser than up the greater curvature.

The accuracy of naked-eye assessment of the extent of the pyloric mucosa was determined in

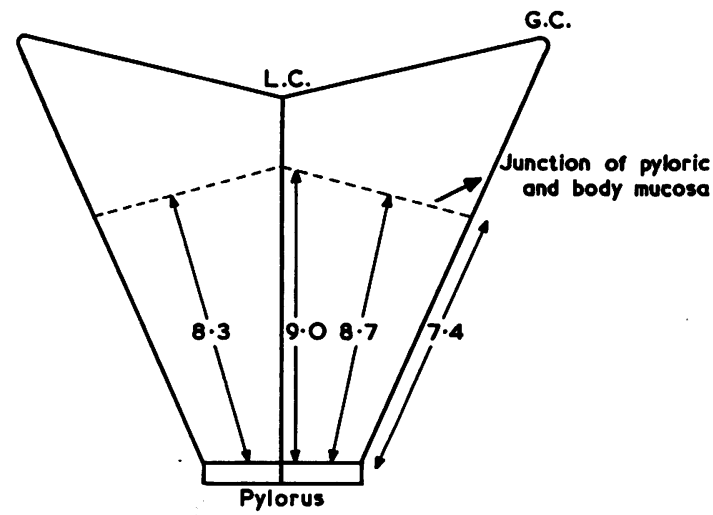

FIG. 1 Plan showing average distribution of pyloric mucosa in partial gastrectomy specimens.

these 18 cases by comparison with the measurements obtained by microscopy (Table II). It was found that naked-eye assessment was reasonably accurate and gave a figure very similar to the correct one, but there was a constant tendency to overestimate the extent of the pyloric mucosa by about $1.0 \mathrm{~cm}$.

TABLE II

NAKED-EYE ASSESSMENT OF PYLORIC MUCOSA ${ }^{1}$

\begin{tabular}{lll} 
& Macroscopic & Microscopic \\
\hline Lesser curve & $9.9 \mathrm{~cm}$. & $9.0 \mathrm{~cm}$. \\
Greater curve & $8.0 \mathrm{~cm}$. & $7.4 \mathrm{~cm}$.
\end{tabular}

${ }^{1}$ Average for 18 cases

STOMAL ULCER Sections were examined from the six additional patients who had a second gastric resection for stomal ulcer following partial gastrectomy and in five pyloric mucosa was found. Sections from four of these cases had been examined in the routine laboratory before the start of this investigation and numerous sections could not be studied, but in each case pyloric mucosa was found. The remaining two cases occurred during the course of this study and numerous sections were cut. The fifth case had a large stomal ulcer, $2.0 \mathrm{~cm}$. in

TABLE I

DUODENAL ULCER IN MALE AND FEMALE CASES

No. Pyloric Mucosa (cm.) Overlap (cm.) Body (cm.) Total Length of Lesser

\begin{tabular}{lrrrrr}
\hline Incomplete removal without overlap & 3 & 9.2 & - & - & 9.2 \\
Incomplete removal with overlap & 2 & 7.5 & 1.9 & - & 9.4 \\
Complete removal without overlap & 26 & 7.2 & - & 3.2 & 10.5 \\
Complete removal with overlap & 32 & 7.1 & 1.0 & 2.7 & 10.8 \\
Average for whole series & 63 & 7.3 & - & - & 10.6
\end{tabular}


diameter, at the junction of gastric and jejunal mucosa and approximately $1.5 \mathrm{sq} . \mathrm{cm}$. of pyloric mucosa was found in the gastric remnant. In the sixth case only a small islet of pyloric mucosa was present but in this patient an abnormally high basal secretion raised the suspicion of the ZollingerEllison syndrome.

GASTRIC ULCER Of the 54 gastrectomy specimens from patients with gastric ulcers, $21(39.0 \%)$ showed pyloric mucosa at the upper cut end of the lesser curvature (Table III).

The type of mucosa in which the ulcer was situated was noted in each case, and it was found that gastric ulcers always developed either within the pyloric mucosa or occasionally at its junction with body mucosa. A gastric ulcer was never seen within the body mucosa. In one case a gastric ulcer was situated in the cardiac glands.

The length of the lesser curve and the extent of the pyloric mucosa along it were related to sex and to the type of ulcer present. The results are shown in Table IV. Although the numbers are too small for statistical analysis, it would appear that in both sexes patients with gastric ulcers have more pyloric mucosa than those with duodenal ulcers. This tendency is more marked in women with gastric ulcers, who have more pyloric mucosa than men with gastric ulcers.

\section{DISCUSSION}

DUODENAL ULCER Of patients who had a partial gastrectomy for duodenal ulcer $7.9 \%$ did not have all the pyloric mucosa removed. We feel that an appreciable amount remained in the gastric remnant in some of these patients for two reasons. First, we have shown that the pyloric mucosa does not extend up the lesser curve in a narrow strip as described by Aschoff (1936), but reaches almost as far along the anterior and posterior walls. This means that any pyloric mucosa remaining in the gastric remnant will extend for some distance on either side of the lesser curve. Secondly, we have found a gradual transition from pyloric to body mucosa in over $50 \%$ of cases. At least some of the cases in which pyloric mucosa is present at the upper cut end of the excised stomach will have a similar overlap, so that the pyloric mucosa may continue for some distance along the lesser curve in the remainder of the stomach.

It is too early to follow up these patients to see if those with pyloric mucosa remaining are more likely to develop stomal ulceration. We feel, however, that it is significant that a considerable amount of pyloric mucosa could be found in each of the four stomachs removed for stomal ulcers which were collected from previous years, although sections had not been taken with the specific intention of looking for it. Continuing production of gastrin by residual pyloric mucosa must be considered as a factor in the production of stomal ulceration in these cases. Pe Thein and Schofield (1962) have found in dogs that only a small amount of pyloric mucosa is sufficient to maintain the hormonal phase of gastric secretion.

It is difficult for the surgeon to be certain of making the line of resection above the upper limit of the pyloric mucosa in view of the wide individual variation. Some surgeons apply a second clamp to remove more of the lesser curve. Taylor (1959) does a modified operation which involves removing a

TABLE III

GASTRIC ULCER IN MALE AND FEMALE CASES No. Pyloric Mucosa (cm.) Overlap (cm.) Body (cm.) $\begin{aligned} & \text { Total Length of Lesser } \\ & \text { Curve (cm.) }\end{aligned}$

\begin{tabular}{lccrr}
\hline Incomplete removal without overlap & 17 & 10.7 & - & - \\
Incomplete removal with overlap & 4 & 10.3 & 0.4 & - \\
Complete removal without overlap & 22 & 8.2 & 10.7 \\
Complete removal with overlap & 11 & 6.9 & 1.4 & 10.3 \\
Average for whole series & 54 & 8.8 & -1.8 & -10.4
\end{tabular}

TABLE IV

AVERAGE LENGTHS OF LESSER CURVE AND OF PYLORIC MUCOSA ALONG THE LESSER CURVE

\begin{tabular}{|c|c|c|c|c|c|c|}
\hline \multicolumn{4}{|c|}{ Duodenal Ulcer } & \multicolumn{3}{|c|}{ Gastric Ulcer } \\
\hline Sex & No. & Lesser Curve $(\mathrm{cm})$. & Pyloric Mucosa (cm.) & No. & Lesser Curve (cm.) & Pyloric Mucosa (cm.) \\
\hline $\mathbf{M}$ & 48 & 10.6 & $7 \cdot 3$ & 43 & $9 \cdot 9$ & $8 \cdot 7$ \\
\hline $\mathbf{F}$ & 15 & $10 \cdot 5$ & $7 \cdot 9$ & 11 & $11 \cdot 7$ & $10 \cdot 1$ \\
\hline
\end{tabular}


much greater length of lesser curve. Naked-eye examination of the stomach immediately after excision will give some indication of whether removal was complete. We found that macroscopic assessment of the extent of the pyloric mucosa was quite accurate and had the additional advantage of tending to overestimate it.

GASTRIC ULCER No gastric ulcers were found in the body mucosa in our series. This agrees with the findings of Magnus (1954) and of Oi, Oshida, and Sugimura (1959).

It is of interest that patients with gastric ulcers tend to have more pyloric mucosa than those with duodenal ulcers. Our figures underestimate this difference, since in $39.0 \%$ of stomachs excised for gastric ulcers, all the pyloric mucosa had not been removed.

The fact that women with gastric ulcers tend to have more pyloric mucosa than men with gastric ulcers is in agreement with the observation of Magnus (1954), who found that $28.7 \%$ of women had a gastric ulcer more than $10 \mathrm{~cm}$. from the pylorus, compared with only $11.8 \%$ of men. The difference in distribution of pyloric mucosa and of gastric ulcers between the sexes also explains the fact that, on average, women with gastric ulcers received a higher gastrectomy than any other patients in the series.

Pyloric mucosa is incompletely removed in a percentage of partial gastrectomies for gastric ulcer, yet stomal ulceration is rare. There are two main reasons for this. Pyloric mucosa in stomachs with gastric ulcers does not produce as much gastrin as in those with duodenal ulcers (Blair, Harper, and
Reed, 1962). Secondly, the augmented histamine test indicates that patients with a gastric ulcer have a smaller parietal cell mass. The fact remains that even a small remnant of pyloric mucosa can maintain the hormonal phase of acid secretion (Pe Thein and Schofield, 1962) and stomal ulcers after partial gastrectomy for duodenal ulcer may be produced in this way.

This work was done during the tenure of grants from the Medical Research Council (A.C.B.D.) and the British Empire Cancer Campaign (M.K.M.) to whom we express our thanks. We are grateful to Mr. H. C. Edwards, C.B.E., Professor H. A. Magnus, and Mr. J. G. Murray for their advice and encouragement.

\section{REFERENCES}

Aschoff, L. (1936). Pathologische Anatomie, 8th ed. Fischer, Jena. Blair, E. L., Harper, A. A., and Reed, J. D. (1962). An assay technique for gastrin. J. Physiol. (Lond.), 163, 47-48P.

Edkins, J. S., and Tweedy, M. (1909). The natural channels of absorption evoking the chemical mechanism of gastric secretion. Ibid., 38, 263-267.

Finsterer, H., and Cunha, F. (1931). The surgical treatment of duodenal ulcer. Surg. Gynec. Obstet., 52, 1099-1114.

Gregory, R. A., and Ivy, A. C. (1941). The humoral stimulation of gastric secretion. Quart. J. exp. Physiol., 31, 111-128.

- and Tracy, H. J. (1961). The preparation and properties of gastrin. J. Physiol., (Lond.), 156, 523-543.

Harper, A. A. (1946). The effect of extracts of gastric and intestinal mucosa on the secretion of $\mathrm{HCl}$ by the cat's stomach. Ibid. $105,31 P$.

Magnus, H. A. (1937). Observations on the presence of intestinal epithelium in the gastric mucosa. J. Path. Bact., 44, 389-398.

- (1954). The pathology of peptic ulceration. Postgrad. med. J., 30, $131-136$

Oi, M., Oshida, K., and Sugimura, S. (1959). The location of gastric ulcer. Gastroenterology, 36, 45-56.

Pe Thein, and Schofield, B. (1962). Biphasic feeding responses in separated fundic pouches in dogs and their relation to the pyloric antrum. Ibid., 43, 436-447.

Taylor, H. (1959). Partial gastrectomy and peptic ulcer. Brit. med.J., 1, 1133-1138. 